\title{
Semiglobal Leader-Following Consensus for generalized homogenous agents
}

\author{
Stefano Battilotti, Claudia Califano
}

\begin{abstract}
In the present paper, the Leader-Following consensus problem is investigated and sufficient conditions are given for the solvability of the problem, assuming that the agents are described by a nonlinear dynamics incrementally homogeneous in the upper bound.
\end{abstract}

Index Terms - Nonlinear systems, Leader-following consensus, stabilization, incremental homogeneity.

\section{INTRODUCTION}

The collective behavior of multi-agent systems is nowadays a topic of great interest due to the wide fields of applications, such as robotics, telecommunications and biology. The literature on the topic considers essentially agents modeled as a linear dynamics (see between the others [14], [16], [17], [23] and the references therein). Different kind of problems have been considered in this context, starting from the general consensus problem where the agents have to reach a common objective, to the leader consensus problem, where the objective of the agents consists in tracking a given dynamics called leader. The dynamics describing the agents may or may not be considered identical. Several results are available in the literature on the leader following problem; we recall between the others [18], [13], [8] where delays are considered, [12], [7], [14], [15] where the topology of the network is switching. Agents described by nonlinear dynamics have been considered for the first time in [6], where the study is restricted to the case in which the leader has a constant trajectory, in [5] where the nonlinearity in the agents dynamics is assumed to be lower triangular and globally Lipschitz and in [3] where the agents dynamics are assumed to be in normal form.

In the present paper the Leader-Following consensus problem is investigated, assuming that the dynamics which describe the agents are incrementally homogeneous in the upper bound as defined in [1]. Such an assumption is a quite general condition for achieving stability by feedback with a guaranteed region of attraction. For the sake of simplicity it is also assumed that the agents are described by the same dynamics. Such a class of systems allows to give sufficient conditions for the solvability of the problem for a large class

Work partially supported by the PON Project PLATINO (Platform for innovative services in future internet)

Dipartimento di Ingegneria Informatica Automatica e Gestionale Antonio Ruberti, Università di Roma La Sapienza, Via Ariosto 25,00185 Italy; battilotti, califano@dis.uniroma1. it of nonlinear systems including lower triangular systems and strict up triangular and many others.

\section{Problem Statement}

Consider a multi agent system consisting of $N$ identical agents and a leader. The leader is governed by the dynamics

$$
\dot{x}^{0}(t)=A x^{0}(t)+f^{0}\left(x^{0}(t)\right)
$$

where for all $t \geq 0, x^{0}(t) \in \mathcal{C} \subset R^{n}, \mathcal{C}$ a compact set containing the origin, whereas each agent is described by the dynamics

$$
\dot{x}^{i}(t)=A x^{i}(t)+B u^{i}(t)+f^{0}\left(x^{i}(t)\right), i \in[1, N]
$$

where $x^{i} \in R^{n}$, and $u^{i} \in R^{m}$ are respectively the state and the control input of the $i$-th agent. We also assume that (2) is controllable in the first approximation around the origin so that $(A, B)$ is assumed without loss of generality to be in Brunowski form.

The information exchange between the $N$ agents and the leader can be represented by means of the graph theory: let $\mathcal{V}=\{1, \cdots, N\}$ be the set of vertices representing the $N$ agents and $\mathcal{E} \subset \mathcal{V} \times \mathcal{V}$ be the set of edges of the graph. An edge of $G$ is denoted by $(i, j)$, representing that agents $\mathrm{i}$ and $\mathrm{j}$ can exchange information between them. The graph is undirected, that is, the edges $(i, j)$ and $(j, i) \in \mathcal{E}$ are considered to be the same. Two nodes $i$ and $j$ are neighbors to each other if $(i, j) \in \mathcal{E}$. The set of neighbors of node $i$ is denoted by $\mathcal{N}_{i}:=\{j \in \mathcal{V}:(j, i) \in \mathcal{E}, j \neq i\}$. A path is a sequence of connected edges in a graph. A graph is connected if there is a path between every pair of vertices. The leader can be represented by a new vertex 0 and information is exchanged between the leader and the agents which are in the neighbors of the leader. Then, the communication exchange is represented by a new graph $\bar{G}$, which consists of graph $G$, vertex 0 and edges between the leader 0 and its neighbors.

The adjacency matrix $Q=\left[q_{i j}\right]$ of a graph $G$ is an $N \times N$ matrix, whose $(i, j)$-th entry is 1 if $(i, j)$ is an edge of $G$ and 0 if it is not. The degree matrix $D$ of $G$ is a diagonal matrix whose $i$-th diagonal element is equal to the cardinality of $\mathcal{N}_{i}$. The Laplacian of $G$ is defined to be a $N \times N$ matrix $L=\left[l_{i j}\right]$ such that $L=-Q+D$. Moreover, $L$ is symmetric if and only if the graph in undirected and $L$ has all its eigenvalues in the closed right half plane and one eigenvalue at zero if and only if the graph is connected. 
In this network, we consider control inputs using local information to make all the $N$ agents follow the leader. In particular, for each agent $i$ the control input is a function of the consensus term

$$
\zeta^{i}(t)=-\sum_{j=1}^{N} \ell_{i j} x^{j}(t)+\ell_{i 0}\left(x^{0}(t)-x^{i}(t)\right)
$$

where the $l_{i j}$ are the entries of the Laplacian of $G$ and $\ell_{i 0}$ is 1 if the leader is a neighbor of agent $i$ and 0 else. The form (3) is motivated by the fact that $-\sum_{j=1}^{N} \ell_{i j} x^{j}(t)=$ $\sum_{j \in \mathcal{N}_{i}}\left(x^{j}(t)-x^{i}(t)\right)$, which means that each agent exchange information only with its neighbors.

Definition 1: The leader-following consensus of system (1)-(2) with consensus term (3) is achieved if the trajectories errors $x^{i}(t)-x^{0}(t)$ of the given system (1)-(2) are bounded for all $t \geq 0$ and $\lim _{t \rightarrow \infty}\left(x^{i}(t)-x^{0}(t)\right)=0$ for all $i \in$ $[1, N]$ and initial conditions $\left(x^{1}(0), \ldots, x^{N}(0)\right) \in \mathcal{C} \times \cdots \times \mathcal{C}$.

Consensus Problem. Consider the multi agent network $(1,2)$ with a fixed communication topology and with full state coupling. Find a state feedback $u^{i}=\alpha\left(\zeta^{i}\right)$ such that the closed-loop system resulting from (1)-(2) achieves the Leader-Following consensus.

\section{RECALLS AND NOTATION}

The following notations are issued from [1].

- $\mathbb{R}^{n}$ (resp. $\mathbb{R}^{n \times n}$ ) is the set of $n$-dimensional real column vectors (resp. $n \times n$ matrices). $\mathbb{R}_{\geq}$(resp. $\mathbb{R}_{\geq}^{n}, \mathbb{R}_{\geq}^{n \times n}$ ) denotes the set of real non-negative numbers (resp. vectors in $\mathbb{R}^{n}$, matrices in $\mathbb{R}^{n \times n}$, with real non-negative entries). $\mathbb{R}_{>}$(resp. $\mathbb{R}_{>}^{n}$ ) denotes the set of real positive numbers (resp. vectors in $\mathbb{R}^{n}$ with real positive entries).

- For any matrix $V \in \mathbb{R}^{p \times n}$ we denote by $V_{i j}$ the $(i, j)$ th entry of $V$ and for any vector $v \in \mathbb{R}^{n}$ we denote by $v_{i}$ the $i$-th element of $v . v_{i}^{k}$ will denote the $i-$ th component of the $k$-th block. We retain a similar notation for functions. For any $v \in \mathbb{R}^{n}$ we denote by $\operatorname{diag}\{v\}$ the diagonal $n \times n$ matrix with diagonal elements $v_{1}, \ldots, v_{n}$. Also, $|a|$ denotes the absolute value of $a \in \mathbb{R},\|a\|$ denotes the Euclidean norm of $a \in \mathbb{R}^{n},\|A\|$ denotes the norm of $A \in \mathbb{R}^{n \times n}$ induced from the Euclidean norm $\|a\|$ and $\langle\langle A\rangle\rangle$ is the matrix obtained from $A$ by substituting each element $a_{i j}$ with its absolute value $\left|a_{i j}\right|$.

- We denote by $C^{0}(\mathbf{X}, \mathbf{Y}), \mathbf{X} \subset \mathbb{R}^{n}$ and $\mathbf{Y} \subset \mathbb{R}^{p}$, the set of continuous functions $f: \mathbf{X} \rightarrow \mathbf{Y}$.

- For any $\epsilon \in \mathbb{R}_{>}$, and for any vectors $x \in \mathbb{R}^{n}, \mathfrak{r} \in \mathbb{R}_{>}^{n}$, we define $\epsilon^{\mathfrak{r}}:=\left(\epsilon^{\mathfrak{r}_{1}}, \cdots, \epsilon^{\mathfrak{r}_{n}}\right)^{T}$ and

$$
\epsilon^{\mathfrak{r}} \diamond x:=\left(\epsilon^{\mathfrak{r}_{1}} x_{1}, \cdots, \epsilon^{\mathfrak{r}_{n}} x_{n}\right)^{T}
$$

that is $\epsilon^{\mathfrak{r}} \diamond x$ is the dilation of a vector $x$ with weights $\mathfrak{r}$. Note that for any $x, y \in \mathbb{R}^{n}, \mathfrak{r}, \overline{\mathfrak{r}} \in \mathbb{R}_{>}^{n}$ and $\epsilon \in \mathbb{R}_{>}$,

$$
\begin{aligned}
\epsilon^{\mathfrak{r}} \diamond \epsilon^{\overline{\mathfrak{r}}} \diamond x & =\epsilon^{\overline{\mathfrak{r}}} \diamond \epsilon^{\mathfrak{r}} \diamond x=\epsilon^{\mathfrak{r}+\overline{\mathfrak{r}}} \diamond x, \\
\left(\epsilon^{\mathfrak{r}} \diamond x\right)^{T}\left(\epsilon^{\overline{\mathfrak{r}}} \diamond y\right) & =\left(\epsilon^{\overline{\mathfrak{r}}} \diamond x\right)^{T}\left(\epsilon^{\mathfrak{r}} \diamond y\right) \\
& =\left(\epsilon^{\mathfrak{r}+\overline{\mathfrak{r}}} \diamond x\right)^{T} y=x^{T}\left(\epsilon^{\mathfrak{r}+\overline{\mathfrak{r}}} \diamond y\right) .
\end{aligned}
$$

To cope with the consensus of nonlinear systems (2) we will use both the Kronecker product notation and a generalized notion of homogeneity recently introduced in [1]. These definitions are recalled below.

Definition 2: (Kronecker product $\otimes$ ). Given a $m \times n$ matrix $A$ and a $p \times q$ matrix $\mathrm{B}$ the Kronecker product $A \otimes B$ is a $P$ matrix of dimension $(m p) \times(n q)$ given by

$$
P=A \otimes B=\left(\begin{array}{ccc}
a_{11} B & \cdots & a_{1 n} B \\
\vdots & \ddots & \vdots \\
a_{m 1} B & \cdots & a_{m n} B
\end{array}\right)
$$

Given a $m$ square invertible matrix $A$ and a $p$ square invertible matrix $B$, then $(A \otimes B)^{-1}=A^{-1} \otimes B^{-1}$. Given the matrices $A, B, C$ and $D$, and assuming that the matrices products $A C$ and $B D$ are defined, then, $(A \otimes B)(C \otimes D)=$ $(A C) \otimes(B D)$.

Denoting by $\mathbf{1}_{m}$ the vector of $\mathbb{R}^{m}$ with all components equal to 1 , then for a fixed $\mathfrak{d} \in \mathbb{R}^{n}, \mathbf{1}_{m} \otimes \mathfrak{d}$ is the vector of $\mathbb{R}^{m n}$ equal to $\left(\mathfrak{d}^{T}, \ldots, \mathfrak{d}^{T}\right)^{T}$. When no confusion is possible the subindex $m$ will be omitted.

Definition 3: (Incremental homogeneity). A function $\phi \in$ $C^{0}\left(\mathbb{R}_{>} \times \mathbb{R}^{n}, \mathbb{R}^{l}\right),(\mu, x) \rightarrow \phi(\mu, x)$, is said to be incrementally homogeneous in the generalized sense (i.h.) with quadruple $(\mathfrak{r}, \mathfrak{e}, \mathfrak{h}, \Phi)$ if there exist $\mathfrak{e} \in \mathbb{R}^{l}, \mathfrak{h} \in \mathbb{R}^{n}, \mathfrak{r} \in \mathbb{R}_{>}^{n}$ and $\Phi \in C^{0}\left(\mathbb{R}^{2 n}, \mathbb{R}^{l \times n}\right)$ such that for all $\epsilon>0$ and $q^{\prime}, q^{\prime \prime} \in \mathbb{R}^{n}$

$\phi\left(\epsilon, \epsilon^{\mathfrak{r}} \diamond q^{\prime}\right)-\phi\left(\epsilon, \epsilon^{\mathfrak{r}} \diamond q^{\prime \prime}\right)=\epsilon^{\mathfrak{e}} \diamond\left(\Phi\left(q^{\prime}, q^{\prime \prime}\right)\left(\epsilon^{\mathfrak{h}} \diamond\left(q^{\prime}-q^{\prime \prime}\right)\right)\right)$

Remark 1: It is worth noting that the function $\phi$ may depend on the dilating parameter itself. As an example, the function $x \rightarrow \phi(x):=x_{1}+x_{2}^{3}$ is i.h. with quadruple $(\mathfrak{r}, 0, \mathfrak{h}, \Phi)$, where $\mathfrak{r}:=(1,2)^{T}, \mathfrak{h}:=(1,6)^{T}$ and $\Phi\left(q^{\prime}, q^{\prime \prime}\right):=\left(1, q_{2}^{\prime 2}+q_{2}^{\prime \prime 2}+q_{2}^{\prime \prime} q_{2}^{\prime}\right)$. The function $\phi(\mu, x)$ which depends on the dilation $\mu:(\mu, x) \rightarrow \phi(\mu, x):=$ $\mu\left(x_{1}+x_{2}^{3}\right), \mu>0$, is i.h. with quadruple $(\mathfrak{r}, 1, \mathfrak{h}, \Phi)$.

Remark 2: Homogeneous systems in the generalized sense (introduced in [1] as a generalization of homogeneous systems in the standard sense ([21])) can be recovered as a particular case of incrementally homogeneous systems in the generalized sense by setting $q^{\prime \prime}=0$ in Definition 3. Moreover, generalized incremental homogeneous systems include large classes of nonlinear systems such as:

i.) locally Lipschitz lower triangular (or norm-bounded by lower triangular maps) $\phi$ :

$$
\phi(x):=\left(\phi_{1}\left(x_{1}\right), \phi_{2}\left(x_{1}, x_{2}\right), \cdots, \phi_{n}\left(x_{1}, \cdots, x_{n}\right)\right)^{T}
$$

where each $\phi_{j}, j=1, \ldots, n$, is (norm-bounded by) a sum of terms of the form $x_{j_{1}}^{t_{j_{1}}} \cdots x_{j_{l}}^{t_{j_{l}}}$ for some reals $t_{j_{i}} \geq 1$. 
ii.) locally Lipschitz strict upper triangular (or normbounded by strict upper triangular maps) $\phi$ :

$$
\phi(x):=\left(\phi_{1}\left(x_{3}, \ldots, x_{n}\right), \cdots, \phi_{n-2}\left(x_{n}\right), 0,0\right)^{T}
$$

where each $\phi_{j}, j=1, \ldots, n-2$, is (norm-bounded by) a sum of terms of the form $x_{j_{1}}^{t_{j_{1}}} \cdots x_{j_{l}}^{t_{j_{l}}}$ for some reals $t_{j_{i}} \geq 1$.

A further generalization can be obtained by noting that there are functions, such as $\phi(x)=\sin x$, which are not i.h. but whose absolute value is bounded by the absolute value of a function which is i.h. This remark motivates the following definition.

Definition 4: A function $\phi \in C^{0}\left(\mathbb{R}_{>} \times \mathbb{R}^{n}, \mathbb{R}^{l}\right),(\mu, x) \rightarrow$ $\phi(\mu, x)$, is said to be incrementally homogeneous in the upper bound in the generalized sense (i.h.u.b.) with quadruple $(\mathfrak{r}, \mathfrak{e}, \mathfrak{h}, \Phi)$ if there exist $\mathfrak{e} \in \mathbb{R}^{l}, \mathfrak{h} \in \mathbb{R}^{n}, \mathfrak{r} \in \mathbb{R}_{>}^{n}$, $\Phi \in C^{0}\left(\mathbb{R}^{2 n}, \mathbb{R}_{\geq}^{l \times n}\right)$ such that for all $\epsilon \geq 1$ and $q^{\prime}, q^{\prime \prime} \in \mathbb{R}^{n}$

$\left\langle\left\langle\phi\left(\epsilon, \epsilon^{\mathfrak{r}} \diamond q^{\prime}\right)-\phi\left(\epsilon, \epsilon^{\mathfrak{r}} \diamond q^{\prime \prime}\right)\right\rangle\right\rangle \preceq \epsilon^{\mathfrak{e}} \diamond\left(\Phi\left(q^{\prime}, q^{\prime \prime}\right)\left\langle\left\langle\epsilon^{\mathfrak{h}} \diamond\left(q^{\prime}-q^{\prime \prime}\right)\right\rangle\right\rangle\right)$ For example, $(\mu, x) \rightarrow \phi(\mu, x):=\mu\left(x_{2} \quad x_{2}^{3} g\left(x_{1}\right)\right)^{T}$, with $g$ any bounded and globally Lipschitz continuous function, is i.h.u.b. with quadruple $(\mathfrak{r}, 1, \mathfrak{h}, \Phi)$, where $\mathfrak{r}:=(1,2)^{T}$, $\mathfrak{e}:=(3,7)^{T}, \mathfrak{h}:=(1,0)^{T}$ and

$\Phi_{11}\left(q^{\prime}, q^{\prime \prime}\right):=0, \Phi_{21}\left(q^{\prime}, q^{\prime \prime}\right):=q_{2}^{\prime \prime 3} \frac{\left|g\left(q_{1}^{\prime}\right)-g\left(q_{1}^{\prime \prime}\right)\right|}{\left|q_{1}^{\prime}-q_{1}^{\prime \prime}\right|}$,

$\Phi_{12}\left(q^{\prime}, q^{\prime \prime}\right):=1, \Phi_{22}\left(q^{\prime}, q^{\prime \prime}\right):=\left|q_{2}^{\prime 2}+q_{2}^{\prime \prime 2}+q_{2}^{\prime} q_{2}^{\prime \prime}\right|\left|g\left(q_{1}^{\prime}\right)\right|$ (note that $q_{2}^{\prime 3} g\left(q_{1}^{\prime}\right)-q_{2}^{\prime \prime 3} g\left(q_{1}^{\prime \prime}\right)=\left(q_{2}^{\prime 3}-q_{2}^{\prime \prime 3}\right) g\left(q_{1}^{\prime}\right)+$ $\left.q_{2}^{\prime \prime 3}\left(g\left(q_{1}^{\prime}\right)-g\left(q_{1}^{\prime \prime}\right)\right)\right)$. The properties of incremental homogeneity (in the generalized sense) are discussed in the Appendix.

\section{Consensus Via State-FEEdBACK}

Let us rewrite the system (1)-(2) in the consensus error coordinates $e^{i}=x^{i}-x^{0}, i \in[1, n]$.

Setting $e=\left(\left(e^{1}\right)^{T}, \cdots,\left(e^{N}\right)^{T}\right)^{T}, \quad u=$ $\left(\left(u^{1}\right)^{T}, \cdots,\left(u^{N}\right)^{T}\right)^{T}$, one thus has

$$
\begin{aligned}
\dot{x}^{0} & =A x^{0}+f^{0}\left(x^{0}\right) \\
\dot{e} & =\left(I_{N} \otimes A\right) e+\left(I_{N} \otimes B\right) u+\hat{F}\left(x^{0}, e\right)
\end{aligned}
$$

where

$$
\hat{F}\left(x^{0}, e\right)=\left(\begin{array}{c}
f^{0}\left(e^{1}+x^{0}\right)-f^{0}\left(x^{0}\right) \\
\vdots \\
f^{0}\left(e^{N}+x^{0}\right)-f^{0}\left(x^{0}\right)
\end{array}\right) .
$$

\section{A. The action of a class of linear change of coordinates}

In the following, we will investigate on the effect of a class of linear change of coordinates on the dynamics (8)(9). More precisely given an invertible $N \times N$ matrix $T$, let us consider the class of change of coordinates

$$
\left(\begin{array}{c}
x^{0} \\
e
\end{array}\right) \rightarrow\left(\begin{array}{c}
x^{0} \\
\tilde{e}
\end{array}\right), \tilde{e}=\left(T \otimes I_{n}\right) e
$$

The peculiarity of this transformation is that it combines $N$ times the $j$-th coordinate of each agent $i$ through the coefficients of each row of $T$, i.e. $\tilde{e}_{i}^{s}=\sum_{j=1}^{N} t_{s, j} e_{i}^{j}$ for $s=i \in[1, N]$ and $i \in[1, n]$. The following result can be stated.

Lemma 1: Let $T$ be an invertible $N \times N$ matrix, and consider the transformation (11). In the new coordinates system (8)-(9) reads

$$
\begin{aligned}
\dot{x}^{0} & =A x^{0}+f^{0}\left(x^{0}\right) \\
\dot{\tilde{e}} & =\left(I_{N} \otimes A\right) \tilde{e}+(T \otimes B) u+\tilde{F}\left(x^{0}, \tilde{e}\right)
\end{aligned}
$$

where $\tilde{F}\left(x^{0}, \tilde{e}\right)=\left(T \otimes I_{n}\right) \hat{F}\left(x^{0},\left(T^{-1} \otimes I_{n}\right) \tilde{e}\right)$.

According to Lemma 3 in the Appendix, since the function $f^{0}\left(x^{0}\right): R^{n} \rightarrow R^{n}$ in (2) is incrementally homogeneous in the upper bound with quadruple $(\mathfrak{r}, \mathfrak{r}+\mathfrak{d}, \mathfrak{h}, \Phi)$, clearly the function $\hat{F}$ defined by (10), is incrementally homogeneous in the upper bound with quadruple

$$
\left(\mathbf{1}_{N+1} \otimes \mathfrak{r}, \mathbf{1}_{N} \otimes(\mathfrak{r}+\mathfrak{d}), \mathbf{1}_{N+1} \otimes \mathfrak{h}, \hat{\Phi}\right)
$$

where setting $p^{i^{\prime}}=e^{i^{\prime}}+x^{0^{\prime}}$ and $p^{i^{\prime \prime}}=e^{i^{\prime \prime}}+x^{0^{\prime \prime}}$, for $i \in[1, N]$, then $\hat{\Phi}\left(\left(e^{\prime}, x^{0^{\prime}}\right),\left(e^{\prime \prime}, x^{0^{\prime \prime}}\right)\right)$ is given by

$$
\hat{\Phi}=\left(\begin{array}{cccc}
\Phi\left(p^{1^{\prime}}, p^{1^{\prime \prime}}\right)+\Phi\left(x^{0^{\prime}}, x^{0^{\prime \prime}}\right) & \Phi\left(p^{1^{\prime}}, p^{1^{\prime \prime}}\right) & 0 & 0 \\
\vdots & 0 & \ddots & 0 \\
\Phi\left(p^{N^{\prime}}, p^{N^{\prime \prime}}\right)+\Phi\left(x^{0^{\prime}}, x^{0^{\prime \prime}}\right) & 0 & 0 & \Phi\left(p^{N^{\prime}}, p^{N^{\prime \prime}}\right)
\end{array}\right)
$$

As it will be shown in the next lemma, under the change of coordinates (11) the function $\hat{F}\left(x^{0}, e\right)$ defined by (10), which is thus incrementally homogeneous with quadruple (13), transforms in the new coordinates into a function $\tilde{F}$ which is still incrementally homogeneous in the upper bound and with the same weights and degrees of $\hat{F}$.

Lemma 2: Assume that the function $f^{0}\left(x^{0}\right): R^{n} \rightarrow R^{n}$ in (2) is incrementally homogeneous in the upper bound in the generalized sense with quadruple $(\mathfrak{r}, \mathfrak{r}+\mathfrak{d}, \mathfrak{h}, \Phi)$. Then under the change of coordinates (11) the function $\tilde{F}\left(x^{0}, \tilde{e}\right): R^{(N+1) n} \rightarrow R^{N n}$ is incrementally homogeneous in the generalized sense in the upper bound with quadruple $(\tilde{\mathfrak{r}}, \tilde{\mathfrak{r}}+\tilde{\mathfrak{d}}, \tilde{\mathfrak{h}}, \tilde{\Phi})$, where $\tilde{\mathfrak{r}}=\mathbf{1}_{N+1} \otimes \mathfrak{r}, \tilde{\mathfrak{d}}=\mathbf{1}_{N} \otimes \mathfrak{d}, \tilde{\mathfrak{h}}=$ $\mathbf{1}_{N+1} \otimes \mathfrak{h}$ and

$$
\tilde{\Phi}\left(\tilde{y}^{\prime}, \tilde{y}^{\prime \prime}\right)=\left(\langle\langle T\rangle\rangle \otimes \max _{\substack{n \\\left\|e^{\prime}\right\| \leq\left\|\left(\langle\langle T-1\rangle\rangle \otimes I_{n}\right) \tilde{e}^{\tilde{e}^{\prime} \|}\\\right\| e^{\prime \prime}\|\leq\|\left(\langle\langle T-1\rangle\rangle \otimes I_{n}\right) \tilde{e}^{\prime \prime} \|}} \hat{\Phi}\left(y^{\prime}, y^{\prime \prime}\right)\right.
$$

with $y^{\prime}=\left(x^{0^{\prime}}, e^{\prime}\right), y^{\prime \prime}=\left(x^{0^{\prime \prime}}, e^{\prime \prime}\right)$ and $\tilde{y}^{\prime}=\left(x^{0^{\prime}}, \tilde{e}^{\prime}\right), \tilde{y}^{\prime \prime}=$ $\left(x^{0^{\prime \prime}}, \tilde{e}^{\prime \prime}\right)$ respectively.

The proof can be obtained by noting that $\tilde{F}\left(x^{0}, \tilde{e}\right)=$ $\varphi_{1}\left(\hat{F}\left(x^{0}, \varphi_{2}(\tilde{e})\right)\right)$ where $\varphi_{1}(\xi):=\left(T \otimes I_{n}\right) \xi$ and $\varphi_{2}(\tilde{e}):=$ $\left(T^{-1} \otimes I_{n}\right) \tilde{e}$, and that for a given $N \times N$ matrix $Q$, then for $\xi \in R^{N n}, \xi \rightarrow \varphi(\xi):=\left(Q \otimes I_{n}\right) \xi$ is incrementally homogeneous in the upper bound in the generalized sense with quadruple $\left(\mathbf{1}_{N} \otimes \mathfrak{r}, \mathbf{1}_{N} \otimes(\mathfrak{r}+\mathfrak{d}),-\mathbf{1}_{N} \otimes \mathfrak{d},\langle\langle Q\rangle\rangle \otimes I_{n}\right)$ where $\mathfrak{d}$ can be chosen in an arbitrary way. 
The result follows through standard computations which make use of the composition rule P2 in the Appendix.

\section{B. The feedback control law}

Consider now the control feedback $u$ for which

$$
u^{j}=\Pi \zeta^{j}=-\Pi\left(\sum_{i=1}^{N} \ell_{j, i} e^{i}+\ell_{j, 0} e^{j}\right) .
$$

for some row vector $\Pi$. Denoting by $L_{0}=$ $\operatorname{diag}\left(l_{1,0}, \cdots, l_{N, 0}\right)$, and by $\hat{L}=L+L_{0}$, in the coordinates $\tilde{e}=\left(T \otimes I_{n}\right) e$, the control reads

$u=-\left(I_{N} \otimes \Pi\right)\left(\hat{L} \otimes I_{n}\right)\left(T^{-1} \otimes I_{n}\right) \tilde{e}=-\left(\hat{L} T^{-1} \otimes \Pi\right) \tilde{e}$.

For the closed-loop system one gets that, setting $\bar{L}=$ $T \hat{L} T^{-1}$

$$
\begin{aligned}
\dot{x}^{0} & =A x^{0}+f^{0}\left(x^{0}\right) \\
\dot{\tilde{e}} & =\left(\left(I_{N} \otimes A\right)-\bar{L} \otimes(B \Pi)\right) \tilde{e}+\tilde{F}\left(x^{0}, \tilde{e}\right),
\end{aligned}
$$

since standard computations show that $(T \otimes$ $B)\left(\left(\hat{L} T^{-1}\right) \otimes K\right)=\bar{L} \otimes(B \Pi)$.

Since $\hat{L}$ is symmetric, it is diagonalizable. We $\operatorname{set} T$ equal to the transformation which diagonalizes $\hat{L}$, i.e.

$$
T \hat{L} T^{-1}=\bar{L}=\operatorname{diag}\left(\bar{\lambda}_{1}, \cdots, \bar{\lambda}_{N}\right) .
$$

In [14], relying upon the fact that each agent is linear (i.e. $f^{0}=0$ ) it is shown that the change of coordinates $\tilde{e}=\left(T \otimes I_{n}\right) e$ is such that (15) consists of $N+1$ independent dynamics, including the leader dynamics. In a nonlinear context this is not true any more as shown in the next lemma. However even if we do not have decoupled dynamics, we still preserve the incremental homogeneity property, which is sufficient for achieving stabilization. In the coordinates (11), where $T$ is the orthogonal transformation which diagonalizes $\hat{L}$, the closed loop system reads

$$
\begin{aligned}
\dot{x}^{0} & =A x^{0}+f^{0}\left(x^{0}\right) \\
\dot{\tilde{e}}^{i} & =\left(A-\bar{\lambda}_{i} B \Pi\right) \tilde{e}^{i}+\tilde{F}^{i}\left(x^{0}, \tilde{e}\right), i=1, \cdots, N .(16)
\end{aligned}
$$

Moreover, as a consequence of Lemma 2, if $f^{0}\left(x^{0}\right)$ is incrementally homogeneous in the generalized sense in the upper bound with quadruple $(\mathfrak{r}, \mathfrak{r}+\mathfrak{d}, \mathfrak{h}, \Phi)$ then

$$
\tilde{F}=\left(\left(\tilde{F}^{1}\right)^{T}, \cdots,\left(\tilde{F}^{N}\right)^{T}\right)^{T}
$$

is incrementally homogeneous in the generalized sense in the upper bound with quadruple $\left(\mathbf{1}_{N+1} \otimes \mathfrak{r}, \mathbf{1}_{N} \otimes(\mathfrak{r}+\mathfrak{d}), \mathbf{1}_{N+1} \otimes\right.$ $\mathfrak{h}, \tilde{\Phi})$ where $\tilde{\Phi}$ is defined in (14).

However, we consider as final system the one obtained from (16) by permuting its states as follows. Let

$$
\begin{aligned}
& \bar{e}_{j}=\left(\tilde{e}_{j}^{1}, \cdots, \tilde{e}_{j}^{N}\right)^{T}, \bar{F}_{j}=\left(\tilde{F}_{j}^{1}, \cdots, \tilde{F}_{j}^{N}\right)^{T} \\
& \bar{e}=\left(\left(\bar{e}_{1}\right)^{T}, \cdots,\left(\bar{e}_{n}\right)^{T}\right)^{T}, \bar{F}=\left(\left(\bar{F}_{1}\right)^{T}, \cdots,\left(\bar{F}_{n}\right)^{T}\right)^{T} \\
& \left.\bar{\Phi}_{j}=\left(\left(\tilde{\Phi}_{j}^{1}\right)^{T}, \cdots,\left(\tilde{\Phi}_{j}^{N}\right)^{T}\right)^{T}, \bar{\Phi}=\left(\left(\bar{\Phi}_{1}\right)^{T}, \cdots,\left(\bar{\Phi}_{n}\right)^{T}\right){ }^{T} 18\right)
\end{aligned}
$$

With this positions, we obtain from (16)

$$
\begin{aligned}
\dot{x}^{0} & =A x^{0}+f^{0}\left(x^{0}\right) \\
\dot{\bar{e}} & =(\bar{A}-\bar{B} \bar{\Pi}) \bar{e}+\bar{F}\left(x^{0}, \bar{e}\right) .
\end{aligned}
$$

with $\bar{A}=A \otimes I_{N}, \quad \bar{B}=B \otimes \bar{\Lambda}, \quad \bar{\Lambda}=$ $\operatorname{diag}\left\{\bar{\lambda}_{1}, \ldots, \bar{\lambda}_{N}\right\}, \bar{\Pi}=\Pi \otimes I_{N}$.

Clearly, if $f^{0}\left(x^{0}\right)$ is incrementally homogeneous in the generalized sense in the upper bound with quadruple $(\mathfrak{r}, \mathfrak{r}+$ $\mathfrak{d}, \mathfrak{h}, \Phi)$ then $\bar{F}$ is incrementally homogeneous in the generalized sense in the upper bound with quadruple $\left(\mathfrak{r} \otimes \mathbf{1}_{N+1},(\mathfrak{r}+\right.$ $\left.\mathfrak{d}) \otimes \mathbf{1}_{N}, \mathfrak{h} \otimes \mathbf{1}_{N+1}, \bar{\Phi}\right)$ where $\bar{\Phi}$ is given in (18).

\section{Achieving consensus}

In this section we will show that there exist a matrix $\bar{\Pi}:=$ $\Pi \otimes I_{N}$ such that the closed-loop system (19) achieves the Leader following consensus. To this aim, we make a basic assumption on the nonlinearity of each agent in terms of incremental homogeneity.

Assumption 1. The nonlinear system (1) is characterized by a function $f^{0} \in C^{0}\left(R^{n}, R^{n}\right)$ incrementally homogeneous in the generalized sense in the upper bound with quadruple $(\mathfrak{r}, \mathfrak{r}+\mathfrak{d}, \mathfrak{h}, \Phi)$ with $\Phi(0,0)=0$ and for $j \in[1, n-1], \mathfrak{h}_{1}:=$ $\mathfrak{d}_{1}, \mathfrak{h}_{j+1}:=\mathfrak{r}_{j+1}-\mathfrak{r}_{j}-\mathfrak{d}_{j}$ and $2 \mathfrak{d}_{j} \leq \mathfrak{r}_{j+1}-\mathfrak{r}_{j} \leq \mathfrak{d}_{j}+\mathfrak{d}_{j+1}$.

Remark 3: In [1] it was proven that Assumption 1 is a sufficient condition to achieve stabilization of $\dot{x}=A x+$ $B u+f^{0}(x)$ where $(A, B)$ are in Brunovski form. The initial assumption that $x^{0}(t) \subset \mathcal{C}$ for all $t \geq 0$, where $\mathcal{C}$ is a compact set containing the origin, is stronger than the one used in [14] ( $A$ is critically stable) and it is introduced to have a bounded behavior of the closed-loop agent trajectories. On the other hand, we allow for more general nonlinearities $f^{0}\left(x^{0}\right)$ 's.

The key Theorem to show achievement of consensus is the following.

Theorem 1: Under Assumptions 1, consider (19) with

$$
\bar{\Pi}=\Pi \otimes I_{N}, \Pi=B^{T} G\left(I_{n}-A^{T} G\right)^{-1}
$$

where $G=\Gamma \operatorname{diag}\left\{\epsilon^{2 \mathfrak{d}_{1}}, \cdots \epsilon^{2 \mathfrak{d}_{n}}\right\}$ and $\Gamma=\operatorname{diag}\left\{\gamma_{1}, \cdots \gamma_{n}\right\}$.

There exist $\epsilon>1$ and $\gamma_{1}, \cdots \gamma_{n}>0$ such that (19) achieves the Leader following consensus.

Proof: Consider the change of coordinates

$$
\left(\begin{array}{c}
x^{0} \\
\bar{e}
\end{array}\right) \rightarrow\left(\begin{array}{c}
x^{0} \\
\hat{e}
\end{array}\right), \hat{e}=\bar{Z}^{-1} \bar{e}
$$

where setting $\bar{G}=G \otimes I_{N}$ and $\bar{\Gamma}=\Gamma \otimes I_{N}$ :

$$
\bar{Z}=I_{n N}-\bar{A}^{T} \bar{G}=\left(I_{n}-A^{T} G\right) \otimes I_{N} .
$$

Due to the particular choice of the change of coordinates it is easily verified that

$$
\dot{\hat{e}}=(\hat{A}-\hat{B} \hat{\Pi}) \hat{e}+\bar{Z}^{-1} \bar{F}\left(x^{0}, \bar{Z} \hat{e}\right)
$$


where $\hat{A}=\bar{Z}^{-1} \bar{A} \bar{Z}, \hat{B}=\bar{Z}^{-1} \bar{B}=\bar{B}, \hat{\Pi}=\bar{\Pi} \bar{Z}=$ $\bar{\Lambda}^{-1} \bar{B}^{T} \bar{G}$.

By noting that $\left(\bar{A}^{T} \bar{G}\right) \bar{A}\left(\bar{A}^{T} \bar{G}\right)=\bar{A}^{T} \bar{G}^{2}$, then, through standard computations one gets that

$$
\bar{Z}^{-1} \bar{A} \bar{Z}=\bar{Z}^{-1}\left(\bar{A}-\bar{A} \bar{A}^{T} \bar{G}=-\bar{A} \bar{A}^{T} \bar{G}+\bar{Z}^{-1}\left(\bar{A}-\bar{A}^{T} \bar{G}^{2}\right)\right.
$$

so that

$$
\begin{aligned}
\hat{A}-\hat{B} \hat{\Pi} & =-\bar{A} \bar{A}^{T} \bar{G}+\bar{Z}^{-1}\left(\bar{A}-\bar{A}^{T} \bar{G}^{2}\right)-\hat{B} \hat{\Pi} \\
& =-\hat{G}+\bar{Z}^{-1}\left(\bar{A}-\bar{A}^{T} \bar{G}^{2}\right)
\end{aligned}
$$

where $\hat{G}:=\operatorname{diag}\left\{I_{N}, \ldots, I_{N}, \bar{\Lambda}\right\} \bar{G}$. As a consequence,

$$
\dot{\hat{e}}=-\hat{G} \hat{e}+\hat{\rho}_{1}(\epsilon, \hat{e})+\hat{\rho}_{2}\left(\epsilon, x^{0}, \hat{e}\right)
$$

where

$$
\begin{aligned}
& \hat{\rho}_{1}(\epsilon, \hat{e})=\left(I_{n N}-\bar{A}^{T} \bar{G}\right)^{-1}\left(\bar{A}-\bar{A}^{T} \bar{G}^{2}\right) \hat{e} \\
& \hat{\rho}_{2}\left(\epsilon, x^{0}, \hat{e}\right)=\left(I_{n N}-\bar{A}^{T} \bar{G}\right)^{-1} \bar{F}\left(x^{0}, \bar{Z} \hat{e}\right) .
\end{aligned}
$$

Let $\hat{\rho}:=\hat{\rho}_{1}+\hat{\rho}_{2}$. Standard computations omitted for space reasons show that $\hat{\rho}$ is incrementally homogeneous in the upper bound in the generalized sense with quadruple $(\mathfrak{r} \otimes$ $\left.\mathbf{1}_{N+1},(\mathfrak{r}+\mathfrak{d}) \otimes \mathbf{1}_{N}, \mathfrak{d} \otimes \mathbf{1}_{N+1},\left(0_{n N \times N}, \hat{R}_{1}\right)+\hat{R}_{2}\right)$, where

$$
\begin{aligned}
& \hat{R}_{1}:=\left(I_{n N}-\bar{A}^{T} \bar{\Gamma}\right)^{-1}\left[\bar{A}+\bar{A}^{T} \bar{\Gamma}^{2}\right], \\
& \hat{R}_{2}\left(q^{\prime}, q^{\prime \prime}\right)=\left(R_{21}\left(q^{\prime}, q^{\prime \prime}\right), \quad \hat{R}_{22}\left(q^{\prime}, q^{\prime \prime}\right)\right) \\
& =\left(I_{n N}-\bar{A}^{T} \bar{\Gamma}\right)^{-1} \hat{\Phi}^{M}\left(q^{\prime}, q^{\prime \prime}\right)\left(\begin{array}{cc}
I_{n} & 0 \\
0 & I_{n N}+\bar{A}^{T} \bar{\Gamma}
\end{array}\right)
\end{aligned}
$$

with $q^{\prime}:=\left(x^{0^{\prime}}, \hat{e}^{\prime}\right), q^{\prime \prime}:=\left(x^{0^{\prime \prime}}, \hat{e}^{\prime \prime}\right)$ and $\hat{\Phi}^{M}$ is any matrix such that all its elements satisfy the inequality

$$
\hat{\Phi}_{i j}^{M}\left(q^{\prime}, q^{\prime \prime}\right) \geq \max _{\substack{\left\|e^{\prime}\right\| \leq\left\|\left(I_{n N}+\bar{A}^{T} \bar{\Gamma}\right) \hat{e}^{\prime}\right\| \\\left\|e^{\prime \prime}\right\| \leq\left\|\left(I_{n N}+\bar{A} T \bar{\Gamma}\right) \hat{e}^{\prime \prime}\right\|}} \bar{\Phi}_{i j}\left(y^{\prime}, y^{\prime \prime}\right)
$$

with $y^{\prime}:=\left(x^{0^{\prime}}, e^{\prime}\right), y^{\prime \prime}:=\left(x^{0^{\prime \prime}}, e^{\prime \prime}\right)$ and $\bar{\Phi}$ is defined in (18).

Since $\hat{\rho}\left(\epsilon, x^{0}, 0\right)=0$, we have as a consequence that setting $y^{\prime}=\left(x^{0}, \hat{e}\right)$ and $y^{\prime \prime}=\left(x^{0}, 0\right)$,

$\left|\hat{\rho}\left(\epsilon, \epsilon^{\mathfrak{r} \otimes \mathbf{1}_{N+1}} \diamond y^{\prime}\right)\right|=\left|\hat{\rho}\left(\epsilon, \epsilon^{\mathfrak{r} \otimes \mathbf{1}_{N+1}} \diamond y^{\prime}\right)-\hat{\rho}\left(\epsilon, \epsilon^{\mathfrak{r} \otimes \mathbf{1}_{N+1}} \diamond y^{\prime \prime}\right)\right|$ $\leq \epsilon^{(\mathfrak{r}+\mathfrak{d}) \otimes \mathbf{1}_{N}} \diamond\left[\hat{R}_{1}\left\langle\left\langle\epsilon^{\mathfrak{d} \otimes \mathbf{1}_{N}} \diamond \hat{e}\right\rangle\right\rangle+\hat{R}_{22}\left(y^{\prime}, y^{\prime \prime}\right)\left\langle\left\langle\epsilon^{\mathfrak{d} \otimes \mathbf{1}_{N}} \diamond \hat{e}\right\rangle\right\rangle\right]$

Moreover, it is easy to see that $(\epsilon, \hat{e}) \rightarrow \hat{G} \hat{e}$ is incrementally homogeneous with quadruple $\left(\mathfrak{r} \otimes \mathbf{1}_{N},(\mathfrak{r}+\mathfrak{d}) \otimes \mathbf{1}_{N}, \mathfrak{d} \otimes\right.$ $\left.\mathbf{1}_{N}, \hat{\Gamma}\right)$ with $\hat{\Gamma}:=\operatorname{diag}\left\{I_{N(n-1)}, \bar{\Lambda}\right\}\left(\Gamma \otimes I_{N}\right)$.

With $V(\hat{e})=\frac{1}{2}\left\|\epsilon^{-\mathfrak{r} \otimes \mathbf{1}_{N}} \diamond \hat{e}\right\|^{2}$ and $e^{\circ}:=\epsilon^{-\mathfrak{r} \otimes \mathbf{1}_{N}} \diamond \hat{e}$ it follows that setting

$$
\begin{aligned}
& \hat{R}(\cdot, \cdot)=\hat{R}_{1}+\hat{R}_{22}\left(\left(\epsilon^{-\mathfrak{r} \otimes \mathbf{1}_{N+1}} \diamond y^{\prime}\right),\left(\epsilon^{-\mathfrak{r} \otimes \mathbf{1}_{N+1}} \diamond y^{\prime \prime}\right),\right. \\
& \dot{V}(\hat{e})=\frac{\partial V}{\partial \hat{e}}(\hat{e})\left\{-\hat{G} \hat{e}+\hat{\rho}\left(\epsilon, x^{0}, \hat{e}\right)\right\} \\
& \left.\leq-\left\langle\left\langle\epsilon^{(\mathfrak{d}-\mathfrak{r}) \otimes \mathbf{1}_{N}} \diamond \hat{e}\right\rangle\right\rangle^{T}[\hat{\Gamma}-\hat{R}(\cdot, \cdot)]\left\langle\left\langle\epsilon^{(\mathfrak{d}-\mathfrak{r}) \otimes \mathbf{1}_{N}} \diamond \hat{e}\right)\right\rangle\right\rangle
\end{aligned}
$$

On account of the fact that $\Phi(0,0)=0$, by construction $\hat{R}_{22}(0,0)=0$. Therefore, for any given $c>0$, there exist $\epsilon^{*}>1$ and $\hat{\Gamma}:=\operatorname{diag}\left\{I_{N(n-1)}, \bar{\Lambda}\right\}\left(\Gamma \otimes I_{N}\right)$ such that

$$
\hat{\Gamma}-\hat{R}(\cdot, \cdot)>0
$$

for all $\hat{e} \in \mathbb{R}^{n N}: V(\hat{e}) \leq c$ and $\epsilon \geq \epsilon^{*}$, in other words $\dot{V}(\hat{e})$ is negative definite over the set $\left\{\hat{e} \in \mathbb{R}^{n N}: V(\hat{e}) \leq c\right\}$. We are left with proving that it is possible to choose $\epsilon \geq \epsilon^{*}$ in such a way that the set $\left\{\bar{e} \in \mathbb{R}^{n N}: V\left(\bar{Z}^{-1} \bar{e}\right) \leq c\right\}$ includes any a priori given compact set $\mathbf{S} \subset \mathbb{R}^{n N}$ for some given $c>0$. Indeed, since $\mathfrak{d}_{1} \leq \mathfrak{d}_{2} \leq \cdots \leq \mathfrak{d}_{n},(\epsilon, \bar{e}) \rightarrow$ $\bar{Z}^{-1} \bar{e}$ is incrementally homogeneous in the upper bound in the generalized sense with quadruple $\left(\mathfrak{r} \otimes \mathbf{1}_{N}, \mathfrak{r} \otimes \mathbf{1}_{N}, \mathbf{0}_{n} \otimes\right.$ $\left.\mathbf{1}_{N},\left(I_{n N}-\bar{A}^{T} \bar{\Gamma}\right)^{-1}\right)$, where $\bar{\Gamma}=\Gamma \otimes I_{N}$. Therefore, for any given compact $\mathbf{S} \subset \mathbb{R}^{n N}$ containing the origin and for any given $c>0$, there exists $\epsilon \geq \epsilon^{*}$ such that

$$
\begin{aligned}
& \max _{\overline{\bar{e}} \in \mathbf{S}} V\left(\bar{Z}^{-1} \bar{e}\right) \leq \max _{\bar{e} \in \mathbf{S}}\left\|\epsilon^{-\mathfrak{r} \otimes \mathbf{1}_{N}} \diamond\left(\left(I_{n N}-\bar{A}^{T} \bar{G}\right)^{-1} \bar{e}\right)\right\|^{2} \\
& \leq \max _{\bar{e} \in \mathbf{S}}\left\|\left(I_{n N}-\bar{A}^{T} \bar{\Gamma}\right)^{-1}\left\langle\left\langle\epsilon^{-\mathfrak{r} \otimes \mathbf{1}_{N}} \diamond \bar{e}\right\rangle\right\rangle\right\|^{2} \leq c .
\end{aligned}
$$

This concludes the proof.

\section{CONCLUSIONS}

In the present paper sufficient conditions have been given for the solvability of the leader following consensus problem on a compact state space, assuming that the dynamics describing the agents are nonlinear and fulfill the property of incremental homogeneity. The study has been performed by assuming a fixed communication topology and full state information. Further research will consider the case in which the topology vary with time, only partial state information is available and will focus also on global aspects. The case in which the dynamics describing the agents are affected by a stochastic noise will be also investigated.

\section{APPENDIX}

\section{A. Properties of incremental homogeneity}

In the following i.h.will denote incremental homogeneous, and i.h.u.b. incremental homogeneous in the upper bound. The following properties are proved in [1].

$P 1$ (addition) For any pair of functions $\phi(\mu, y), \psi(\mu, y) \in$ $C^{0}\left(\mathbb{R}_{>} \times \mathbb{R}^{n}, \mathbb{R}^{l}\right)$, i.h.u.b. (resp. i.h.) with quadruple resp. $(\mathfrak{r}, \mathfrak{e}, \mathfrak{h}, \Phi)$ and $(\mathfrak{r}, \mathfrak{e}, \mathfrak{h}, \Psi)$, the function $(\mu, y) \rightarrow \phi(\mu, y)+$ $\psi(\mu, y)$ is i.h.u.b. (resp. i.h.) with quadruple $(\mathfrak{r}, \mathfrak{e}, \mathfrak{h}, \Phi+\Psi)$.

P2 (composition) For any i.h.u.b. functions $\phi \in C^{0}\left(\mathbb{R}_{>} \times\right.$ $\left.\mathbb{R}^{s}, \mathbb{R}^{l}\right),(\mu, y) \rightarrow \phi(\mu, y)$, with quadruple $(\mathfrak{r}, \mathfrak{e}, \mathfrak{h}, \Phi)$ and $\psi \in$ $C^{0}\left(\mathbb{R}_{>} \times \mathbb{R}^{n}, \mathbb{R}^{s}\right),(\mu, x) \rightarrow \psi(\mu, x)$, with quadruple $(\mathfrak{r},-\mathfrak{h}+$ $\mathfrak{r}, \mathfrak{p}, \Psi)$ if there exists $\Phi^{M} \in C^{0}\left(\mathbb{R}^{2 s}, \mathbb{R}_{\geq}^{l \times s}\right)$ such that for all $\epsilon \geq 1$ and $q^{\prime}, q^{\prime \prime} \in \mathbb{R}^{n}$

$$
\Phi\left(\epsilon^{-\mathfrak{r}} \diamond \psi\left(\epsilon^{\mathfrak{r}} \diamond q^{\prime}\right), \epsilon^{-\mathfrak{r}} \diamond \psi\left(\epsilon^{\mathfrak{r}} \diamond q^{\prime \prime}\right)\right) \preceq \Phi^{M}\left(q^{\prime}, q^{\prime \prime}\right)
$$


then $(\mu, x) \rightarrow \phi(\mu, \psi(\mu, x))$ is i.h.u.b. with quadruple $\left(\mathfrak{r}, \mathfrak{e}, \mathfrak{p}, \Phi^{M} \Psi\right)$.

Remark 4: In particular, for $\phi$ with constant $\Phi$ (23) is trivially satisfied with $\Phi^{M}=\Phi$ and $(\mu, x) \rightarrow \phi(\mu, \psi(\mu, x))$ is i.h.u.b. with quadruple $(\mathfrak{r}, \mathfrak{e}, \mathfrak{p}, \Phi \Psi)$.

Let $(A, B)$ be in Brunowski canonical form. Note that $A^{T}$ is the Moore-Penrose pseudoinverse of $A$, that is $A^{T} A A^{T}=$ $A^{T}, A A^{T} A=A,\left(A^{T} A\right)^{T}=A^{T} A$ and $\left(A A^{T}\right)^{T}=$ $A A^{T}$. Therefore $I-A A^{T}$ is the orthogonal projection onto $(\operatorname{Im}\{A\})^{\perp}=\operatorname{Im}\left\{I-A A^{T}\right\},(\operatorname{Im}\{W\}$ denotes the vector space generated by the columns of the matrix $W$ ). Then:

$P 3.1$ (shifting I) for any $\mathfrak{z} \in \operatorname{Im}\left\{I-A A^{T}\right\}$ and any function $\phi \in C^{0}\left(\mathbb{R}_{>} \times \mathbb{R}^{n}, \mathbb{R}^{l}\right):(\mu, x) \rightarrow \phi(\mu, x)$, i.h.u.b. (resp.i.h.) with quadruple $(\mathfrak{r}, \mathfrak{e}, \mathfrak{h}, \Phi)$, the function $(\mu, x) \rightarrow A \phi(\mu, x)$ is i.h.u.b. (resp.i.h.) with quadruple $(\mathfrak{r}, A \mathfrak{e}+\mathfrak{z}, \mathfrak{h}, A \Phi)$

P3.2 (shifting II) for any $\mathfrak{z} \in \operatorname{Im}\left\{I-A^{T} A\right\}$ and any function $\phi \in C^{0}\left(\mathbb{R}_{>} \times \mathbb{R}^{n}, \mathbb{R}^{l}\right):(\mu, x) \rightarrow \phi(\mu, x)$, i.h.u.b. (resp.i.h.) with quadruple $(\mathfrak{r}, \mathfrak{e}, \mathfrak{h}, \Phi)$ and constant $\Phi$, the function $(\mu, x) \rightarrow \phi(\mu, A x)$ is i.h.u.b. (resp.i.h.) with quadruple $\left(\mathfrak{r}, \mathfrak{e}, A^{T}(\mathfrak{h}-\mathfrak{r})+\mathfrak{r}+\mathfrak{z}, \Phi A\right)$.

\section{B. Additional results}

The proofs of the next results are omitted for space reasons.

Lemma 3: Let $\varphi(q): R^{n} \rightarrow R^{n}$ be a function incrementally homogeneous in the upper bound with quadruple $\left(\mathfrak{r}, \mathfrak{r}+\mathfrak{d}, \mathfrak{h}, \Phi\left(q^{\prime}, q^{\prime \prime}\right)\right)$, then the function $\hat{\varphi}(q, \omega)=\varphi(q+$ $\omega)-\varphi(q): R^{2 n} \rightarrow R^{n}$ is incrementally homogeneous in the upper bound with quadruple $(\mathfrak{r}, \mathfrak{r}+\mathfrak{d}, \mathfrak{h}, \hat{\Phi})$, with

$\hat{\Phi}=\left(\Phi\left(q^{\prime}+\omega^{\prime}, q^{\prime \prime}+\omega^{\prime \prime}\right)+\Phi\left(q^{\prime}, q^{\prime \prime}\right), \Phi\left(q^{\prime}+\omega^{\prime}, q^{\prime \prime}+\omega^{\prime \prime}\right)\right)$

Lemma 4: Let $\varphi(q): R^{n} \rightarrow R^{n}$ be a function i.h.u.b. with quadruple $\left(\mathfrak{r}, \mathfrak{r}+\mathfrak{d}, \mathfrak{h}, \Phi\left(q^{\prime}, q^{\prime \prime}\right)\right)$, then the function $\hat{\varphi}$ : $(\epsilon, q) \rightarrow \varphi\left(\sigma\left(\beta \epsilon^{\mathfrak{r}}, q\right)\right)$ is i.h.u.b. with quadruple $(\mathfrak{r}, \mathfrak{r}+$ $\left.\mathfrak{o}, \mathfrak{h}, \Phi^{0}\right)$, where $\Phi^{0}$ is a constant matrix such that $\forall q^{\prime}, q^{\prime \prime}$ : $\left\|q^{\prime}\right\|,\left\|q^{\prime \prime}\right\| \leq n \beta$

$$
\Phi\left(q^{\prime}, q^{\prime \prime}\right) \preceq \Phi^{0}
$$

\section{REFERENCES}

[1] S. Battilotti, Incremental generalized homogeneity, observer design and semi global stabilization, Asian Journal of Control,16, 498-508, 2014.

[2] A. Bacciotti, L. Rosier, Lyapunov functions and stability in control theory, LNCS, 267, Springer, Berlin, 2001.

[3] F. Delli Priscoli, A. Isidori, L. Marconi, A. Pietrabissa, Leaderfollowing coordination of nonlinear agents under time-varying communication topologies, IEEE 53rd Conference on Decision and Control, 797-804, 2014

[4] A. Fax, R. M. Murray,Information flow and cooperative control of vehicle formations, IEEE Trans. on Automatic Control,49, 1465 -1476, 2004.

[5] H.F. Grip, A. Saberi, A.A. Stoorvogel, Synchronization in Networks of Nonlinear, Non-Introspective, Minimum-Phase Agents without Exchange of Controller States, ACC 2014, Portland, USA, 2014

[6] L. Guo, X. Nian, H. Pan, Leader-following consensus of multi-agent systems with switching topologies and time-delays, J. Control Theory Appl, 11, 306-310, 2013
[7] Y. Hong, G. Chen, L. Bushnell, Distributed observers design for leaderfollowing control of multi-agent networks, Automat., 44, 846- 850, 2008.

[8] Y. Hong, J. Hu, L. Gao,Tracking control for multi-agent consensus with an active leader and variable topology, Automat.,42, 1177-1182, 2006.

[9] J. Hu, G. Feng. Distributed tracking control of leader-follower multiagent systems under noisy measurement, Automat., 46, 1382-1387, 2010.

[10] J. Hu, Y. Hong,Leader-following coordination of multi-agent systems with coupling time delays, Physica A, 374, 853-863, 2007.

[11] Z. Lin, B. Francis, M. Maggiore,Necessary and sufficient graphical conditions for formation control of unicycles, IEEE Trans. on Automatic Control, 50, 121-127, 2005.

[12] B. Liu, T. Chu, L. Wang, et al.,Controllability of a leader-follower dynamic network with switching topology, IEEE Trans. on Automatic Control, 53, 1009-1013, 2008.

[13] Z. Meng, W. Ren, Y. Cao, et al.,Leaderless and leader-following consensus with communication and input delays under a directed network topology, IEEE Trans. on Systems, Man, and Cybernetics Part B: Cybernetics, 41: 75-88, 2011.

[14] W. Ni, D. Cheng, Leader-following consensus of multi-agent systems under fixed and switching topologies, Sys. Cont. Let., 59, 209-217, 2010.

[15] G. Notarstefano, M. Egerstedt, M. Haque,Containment in leaderfollower networks with switching communication topologies, Autom.,47, 1035-1040, 2011.

[16] R. Olfati-Saber, R.M. Murray, Consensus problems in networks of agents with switching topology and time-delays, IEEE Trans. on Automatic Control, 49, 1520-1533, 2004.

[17] R. Olfati-Saber,J. Alex Fax, R.M. Murray,Consensus and Cooperation in Networked Multi-Agent Systems Proc. of the IEEE, 95, 215-233, 2007

[18] K. Peng, Y. Yang,Leader-following consensus problem with a varyingvelocity leader and time-varying delays, Physica A,388 193-208, 2009.

[19] W. Ren. Multi-vehicle consensus with a time-varying reference state, Sys. Cont. Lett., 56, 474-483, 2007.

[20] W. Ren, R.W. Beard, E.M. Atkins, A Survey of Consensus Problems in Multi-agent Coordination, American Cont. Conf., Portland (USA), 1859-1864, 2005.

[21] L. Rosier, Homogeneous Lyapunov function for homogeneous continuous vector field, Syst. Contr. Lett., 19, 467-473, 1992.

[22] G. Shi, Y. Hong,Global target aggregation and state agreement of nonlinear multi-agent systems with switching topologies, Automat.,45, $1165-1175,2009$

[23] J. Wang, D. Cheng, $\mathrm{X}$. Hu, Consensus of multi-agent linear dynamical systems, Asian Journal of Control 10, 144-155, 2008. 